\title{
An engineering approach for the fast simulation of radial inflow turbines with vaneless spiral casing by single-channel CFD models
}

\author{
Piero Danieli ${ }^{1}$, Massimo Masi $^{2}$, Andrea Lazzaretto ${ }^{1}$, Gianluca Carraro ${ }^{1}$ \\ ${ }^{1}$ University of Padova, Department of Industrial Engineering - DII - Padova, Italy \\ ${ }^{2}$ University of Padova, Department of Management and Engineering - DTG - Vicenza, Italy
}

\begin{abstract}
The basic RANS-CFD analysis of the simplest radial-inflow turbine configuration is the subject of this paper. An original technique is here proposed to model the effect of the vaneless spiral casing using single-channel CFD calculations and providing an effective alternative to the more complex simulation of the 360-degree domain otherwise required to simulate this turbine configuration. The aim of the paper is to verify the effectiveness of the proposed modelling technique as a reliable engineering approach conceived to support the preliminary design phase of radial-inflow turbines with time-effective CFD calculations. To this end, the open-source CFD code MULTALL has been used to predict the aerodynamic performance of optimal designs of radial-inflow turbines with different specific speed and diameter and working with air as ideal gas. The MULTALL predictions are compared with the corresponding steady-state results obtained by calculations suited to the preliminary assessment of radial turbines designs performed on fully 360-degree

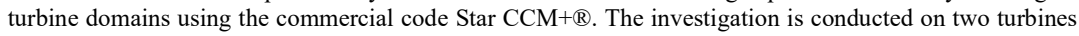
that are designed in accordance with a widely validated method. The results show that the proposed CFD approach predicts well the trends and values of the aerodynamic performance of both the turbine designs: a $5 \%$ overestimation of the performance predicted by the fully 360 -degree CFD models was never exceeded. The suggested turbine modelling approach implemented in MULTALL requires a three times lower computation time than the corresponding traditional 360-degree model.
\end{abstract}

Keywords: CFD, radial turbines, preliminary design of turbomachinery, Multall

\section{Introduction}

The most complex configuration of the radial inflow turbine includes upstream of the runner: a), a spiral casing - to convey the flow towards the entire arc of admission of the runner and obtain an even distribution of the mass flow rate in all the rotating channels; and b), a vaned radial nozzle - providing to the flow both the velocity magnitude and direction required by the proper operation of the runner (see e.g. [1]). A simpler and more compact configuration is composed only of a vaneless spiral casing to carry out both the previous tasks a) and b) [1]. As per other turbomachine types, Computational Fluid Dynamics (CFD) has become by decades a powerful tool to analyze both the global aerodynamic performance and the details of the local flow field in radial turbines [2,3], as well as to support their design and optimization, allowing a noticeable reduction of the costs related to the experimental testing. Focusing on the design process, single-channel CFD calculations are widely used to support the preliminary phase where quick and computationally low-cost predictions of the global aerodynamic performance are preferred to more accurate (and more computationally demanding) solutions of the fully $360^{\circ}$ fluid domain. Single-channel calculations are commonly applied to radial inflow turbines with nozzle vanes (in which the spiral casing can be excluded from the domain) by changing the actual numbers of fixed and/or moving blades to obtain a domain periodicity with reduced azimuthal width [4]. These simplified simulations rely on the Multi-Reference-Frame (MRF) approach that solves the RANS equations in the relative reference frames (i.e., steady or rotating for fixed and moving blade rows, respectively). MRF allows performing steadystate calculations by adding the Coriolis and centrifugal contributions in the momentum equations. In fact, these two terms arise when the flow field within the rotating domain is described by means of equations valid for a reference frame moving with the domain itself [5]. Different interface techniques are used in conjunction with MRF [5]: the mixing-plane interface performs some circumferential averaging of the fluid-dynamic variables keeping the local distribution along the 
blade span direction; the frozen-rotor interface saves the local distribution of the variables across the interface keeping the moving blades "frozen" at a specific position relative to the fixed blades. Single-channel simulations cannot support the design of radial turbines with vaneless spiral casing because the lack of the nozzle vanes does not allow to satisfy the domain periodicity requirement. To overcome this limitation, the authors propose here a novel approach to convert the geometric features of an actual vaneless spiral casing to a corresponding geometry compatible with the capabilities of single-channel CFD models. The results obtained using this approach are compared with those obtained by simulations of the entire turbine flow domain extending from the casing throat to the runner discharge for two radial turbine designs to investigate on the limitations of the suggested approach. The simulations of the fully $360^{\circ}$ turbine domains were performed using the state-of-the-art CFD code Siemens Star CCM+®, whereas the simulations of the single-channel domains were performed using the open-source code MULTALL, which was specifically conceived for steady-state single-passage calculations. MULTALL has been developed by John Denton since the ' 70 when it was used for twodimensional modelling of steam and gas turbines [6]. Over the years, the code has been modified and updated following the improvement of the computing technologies. Accordingly, multi-grid-techniques, non-overlapping grids, fully 3D simulations, and mixing plane approach were progressively implemented in the code [7-10]. Finally, MULTALL has been made available as an open source code in 2017 [11]. Many works can be found in the literature proving the reliability and applicability of the code to different types of high-speed turbomachines including the tip leakage analysis [12-18]. Recently, the present authors demonstrated that the use of MULTALL can be profitably extended also to support the design of low-speed axial-flow fans [19]. The selection of MULTALL as CFD code to support the application of the novel modelling approach presented in this work was driven by the positive results obtained by [19] and by the present incapability of MULTALL to simulate vaneless spiral casings. Accordingly, the aim of the paper is twofold:

i) validating the proposed modelling approach;

ii) extending the use of MULTALL as a reliable tool to support also the preliminary design of radial-inflowturbines.

\section{Vaneless spiral casing model for single-channel CFD}

In accordance with the azimuthal periodicity of a turbine runner with $\mathrm{z}$ blades, only a $360 \% \mathrm{z}$ sector of the spiral casing can be included in the geometrical domain of a single-channel steady-state CFD calculation. An axis-symmetric radial Transition Piece (TP) is suggested to model the original vaneless spiral casing and satisfy the periodicity requirement of single-channel calculations. Figure 1 sketches the meridional section (Fig. 1a) and the circumferential view (Fig. 1b) of a radial-inflow turbine single-blade passage including the spiral casing model (see the dashed circles) proposed by the authors. The air flow enters the domain through the outermost cylindrical surface and one of the two side surfaces, which define the azimuthal boundaries of the TP. The other side surface is crossed by the air flow that the real vaneless spiral casing would have directed towards the successive blade channels of the runner. The imposition of the periodic condition to these side surfaces therefore allows to convey the flow towards the entire arc of admission of the runner. Note that, the periodicity of the domain also imposes that velocity and pressure distributions of the flow approaching the runner are the same for all the blade passages.

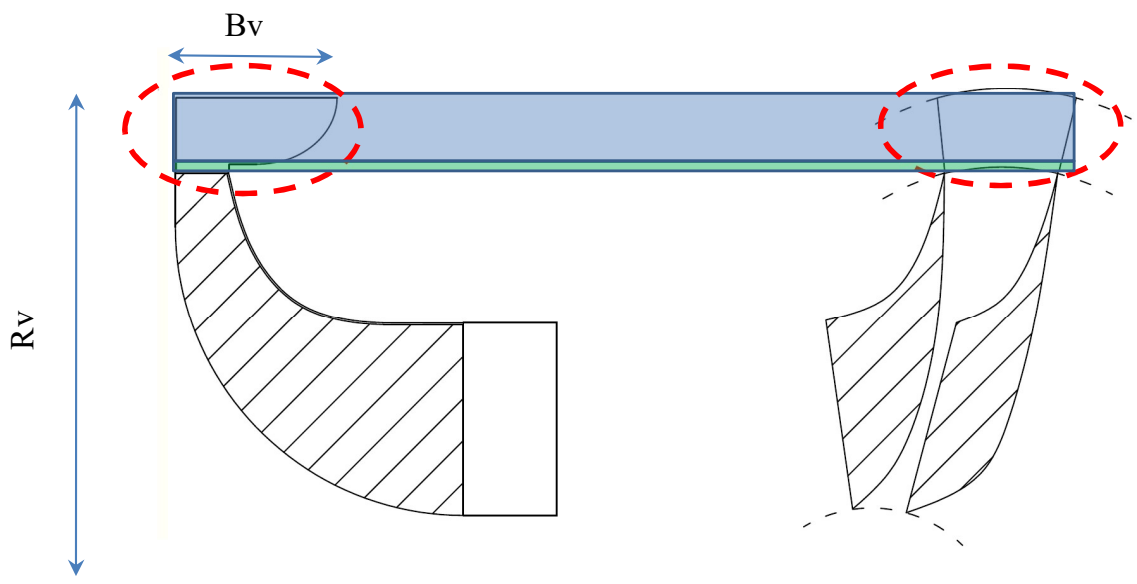

a)

b)

Fig. 1 - Single blade passage domain radial-inflow turbines: sketches of the meridional section a)-and circumferential b) views. TP is circled in red.

The conceptual basis under the validity of the proposed modelling approach is the definition of a series of equivalence constraints ensuring that the flow field across the runner fed by the TP is equal to the corresponding flow field in the 
actual radial inflow turbine. The equivalence of the flow fields developing on two domains (the radial inflow turbine and its single-channel model counterpart) that are not geometrically similar in the corresponding inlet regions (i.e., the spiral casing and the TP) can be achieved when the geometrically similar parts of the two domains (i.e., the turbine runners) feature:
a. kinematic equivalence (i.e., velocity vectors);
b. dynamic equivalence (i.e., blade load);
c. total pressure losses equivalence (i.e., friction/viscous effects in the region upstream of the runner).

The first of the three following sub-Sections focuses on the geometry constraints needed to fulfill the item c, whereas the second sub-Section deals with the boundary conditions required to fulfill the items $a$ and $b$. Finally, the third sub-Section states the major theoretical limitation of the proposed modelling approach.

\subsection{Total pressure losses equivalence constraint}

Losses due to wall friction in a pipe depend on surface finishing, fluid velocity, mass density, and hydraulic diameter. If the surface friction factor, fluid velocity and mass density have uniform azimuthal distribution along the spiral casing, the total pressure losses per unit length change as a function of the casing azimuth angle $(\vartheta)$ - measured from the inlet throat - because the hydraulic diameter of the spiral casing cross section decreases as $\vartheta$ increases. Accordingly, the friction losses should be maximum at the end $\left(\vartheta=360^{\circ}\right)$ and minimum at the beginning $\left(\vartheta=0^{\circ}\right)$ of the vaneless spiral casing. This behavior of the losses can be modelled correctly only including the full geometry of the spiral casing in the computational domain. However, a reasonable averaging is expected by a periodic model in which the innermost part of the TP (i.e., the green colored part in Fig. 1, which mimics the vaneless nozzle) perfectly copies the exit zone of the actual vaneless spiral casing, whereas the outermost part (i.e., the blue colored part in Fig.1, which mimics the spiral volute) features radius $(\mathrm{Rv})$ and width $(\mathrm{Bv})$ equal to some averaged values of the corresponding parameters in the cross-section of the actual spiral casing. Given that Rv and Bv have almost linear dependence on $\vartheta$, they are kept equal to the local values at half azimuth of the actual spiral casing $\left(\vartheta=180^{\circ}\right)$.

\subsection{Dynamics/kinematics equivalence constraints}

Figure 2 shows the detail of the circumferential section of the TP and the rectified view of the domain exit region sketched in Fig. 1b) and illustrates the set of boundary conditions required by the proposed modelling approach.

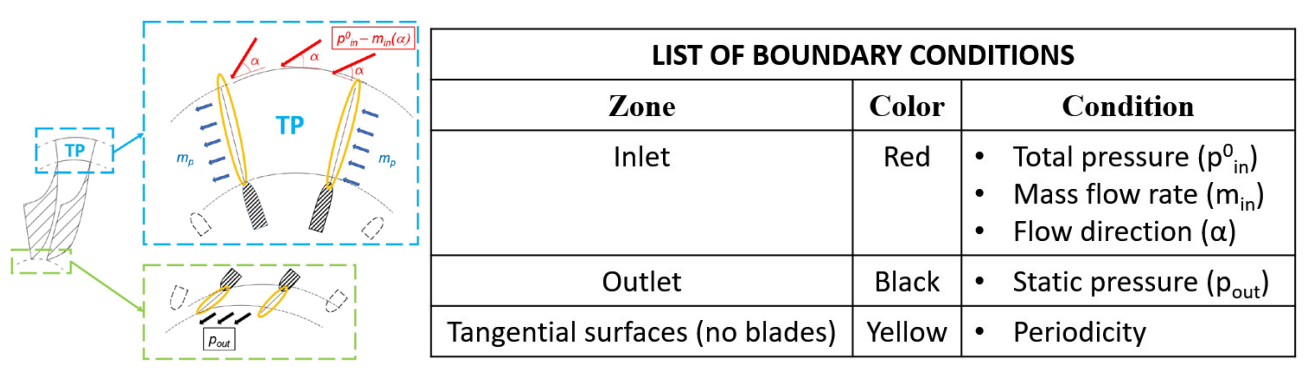

Fig. 2 - Physic boundaries and corresponding boundary conditions.

In particular, Total-pressure-inlet and static-pressure-outlet applied at the outermost cylindrical surface and downstream of the runner, respectively, are the boundary conditions that allow fulfilling the turbine pressure ratio $\left(r_{p}\right)$. Note that, at fixed rotational speed, the imposition of the pressure boundary condition in the single-channel model is necessary but not sufficient to ensure the flow field of the corresponding 360-degree turbine model, because the shapes of the two domains are not identical. The addition of the fixed flow direction ( $\alpha$ in Fig. 2) boundary condition at the total-pressure inlet boundary is therefore required to fulfill both the dynamic and kinematic equivalence. In fact, the specification of the flow direction at fixed pressure drop and rotational speed permits to set the mass flow rate crossing the periodic boundaries of the TP $\left(m_{p}\right)$ to the value consistent with the mass flow across the entire turbine, i.e. $z$-times the mass flow rate across the blade passage $\left(m_{i n}\right)$. Assuming an almost linear decrease of the cross-sectional area of the spiral casing with the azimuth angle, an effective way to find the unique value admissible for the flow direction $\alpha$ is to check the fulfillment of the following equation: 


$$
\frac{A_{p}}{A_{\text {in }}}=\frac{m_{p}}{m_{\text {in }} * Z}
$$

where $A_{p}\left[\mathrm{~m}^{2}\right]$ is the area of one periodic surface of the TP and $A_{i n}\left[\mathrm{~m}^{2}\right]$ is the cross-section area at the throat of the real spiral casing. The graphical explanation of Eq. (1) is reported in Tab.1 considering four TP sections corresponding to the azimuth angles $0^{\circ}, 90^{\circ}, 180^{\circ}$ and $270^{\circ}$.

Tab.1 - Graphical explanation of Eq. (1)

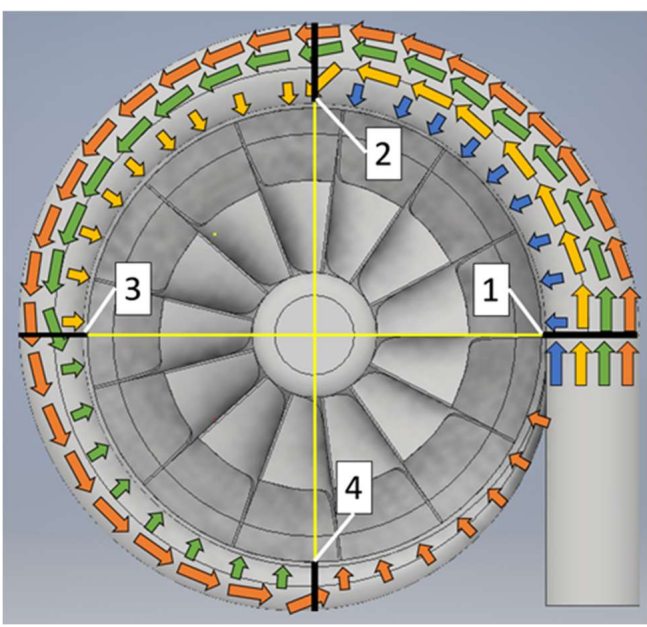

\begin{tabular}{|c|c|c|}
\hline $\begin{array}{c}\text { Spiral } \\
\text { casing } \\
\text { section }\end{array}$ & $A_{p}$ & $m_{p}$ \\
\hline 1 & $=A_{\text {in }}$ & $\begin{array}{c}=m_{\text {tot }}=m_{\text {in }} * Z \\
\left(1^{\text {st }}+2^{\text {nd }}+3^{\text {rd }}+4^{\text {th }}\right. \\
\text { quarters })\end{array}$ \\
\hline 2 & $=0.75 * A_{\text {in }}$ & $\begin{array}{c}=0.75 * m_{\text {tot }} \\
\left(2^{\text {nd }}+3^{\text {rd }}+4^{\text {th }} \text { quarters }\right)\end{array}$ \\
\hline 3 & $=0.5 * A_{\text {in }}$ & $\begin{array}{c}\left(3^{\text {rd }}+4^{\text {th }} \text { quarters }\right) \\
=0.5 * m_{\text {tot }}\end{array}$ \\
\hline 4 & $=0.25 * A_{\text {in }}$ & $\begin{array}{c}\left(^{\text {th }} \text { quarter }\right) \\
\text { tot }\end{array}$ \\
\hline
\end{tabular}

\subsection{Theoretical limitations of the model}

The three equivalence constraints formalised above rely on three major assumptions:

A. The outer part of the real casing (i.e., the volute) should assure uniform distribution of the flow in each cross section, and even distribution of the flow rate in the runner vanes;

B. The inner part of the casing (i.e., the "nozzle-like" annulus) should assure the proper magnitude and direction of the exit velocity;

C. The average value of the product between velocity and mass density remains the same in each cross section of the spiral casing, as stated in Eq. (1).

Accordingly, the higher the departure of the volute design from these assumptions the lower the theoretical reliability of the proposed model. Furthermore, given that the proposed spiral casing modelling approach does not include the real geometry of the volute, 3D flow phenomena and turbulent viscosity distribution strictly related to the local details of the geometry (e.g. the volute tongue) are unavoidably neglected.

\section{Method}

The vaneless spiral casing modelling approach has been applied to two radial inflow turbine test cases, subject of the first following sub-section. The second sub-section defines the parameters used to quantify the performance of these turbine designs and to perform the comparisons presented in the paper.

\subsection{Radial turbine test cases}

The two turbines with vaneless spiral casing have been designed with the preliminary design procedure proposed by Whitfield et al. [20]. Focusing on the design of the casing, the simplest model commonly used to design this component relies on the one-dimensional flow hypothesis. This model considers only the tangential and radial velocity components and neglects both the secondary flows due to the shape of the cross-section area and the recirculating flows in the region surrounding the volute tongue. Accordingly, under the assumption of angular momentum conservation, a free vortex flow 
centred in the rotation axis is expected. The standard one-dimensional model commonly includes the assumption of incompressible flow that does not allow to take into account the density variation that occurs within the volute. For this reason, Whitfield et al. [20] suggest the use of the compressible-free vortex approach proposed by Chappie et al. [22], who demonstrated that radial turbines with spiral casings designed in accordance with their approach perform better than those having casings designed using to the incompressible method.

The two turbine designs have been conceived to investigate if the vaneless spiral casing modelling offers a good degree of accuracy within a range of optimal turbine shapes. The characteristics of the two designs are described in the following while their design parameters are listed in Tab.2:

- "NSO5" machine: overall optimal design

This turbine has been designed to match the optimal design specifications suggested by [20] that lead to a machine with specific speed $\left(n_{s}\right)$ and specific diameter $\left(d_{s}\right)$ equal to 0.56 and 3.6, respectively. The NS05 design fulfills these dimensionless parameters using the total inlet pressure $p_{\text {oin }}$, the static outlet pressure $p_{\text {out }}$ and the rotational speed $R P M$ as design specifications.

- "NSO3" machine: constrained optimal design

Compared to the NS05, this turbine has been designed fixing the mass-flowrate $m_{i n}$ as an additional constraint. Accordingly, the absolute optimal $n_{s}$ - $d_{s}$ pair chosen for the NS03 design (equal to 0.35 and 5.38, respectively) still belongs to the optimum Cordier line suggested in [22].

Tab. 2 - Design parameters of the considered turbomachines

\begin{tabular}{|c|c|c|c|}
\hline & Design ID & NS05 & NS03 \\
\hline Turbine component & Design parameter & \multicolumn{2}{|c|}{ value } \\
\hline \multirow{19}{*}{ Runner } & Specific speed, $n_{s}[-]$ & 0.56 & 0.35 \\
\hline & Specific diameter, $d_{s}[-]$ & 3.62 & 5.38 \\
\hline & Rotational speed, $R P M$ [rev/min] & 60000 & 100000 \\
\hline & Tip speed ratio, $u / C_{0}[-]$ & 0.72 & 0.67 \\
\hline & Absolute inlet total pressure, $P_{\text {in }}[\mathrm{bar}]$ & 8.01 & 1.61 \\
\hline & Absolute outlet static pressure, $P_{\text {out }}[$ bar $]$ & 2.01 & 1.03 \\
\hline & Inlet absolute flow angle, $\alpha_{2}[\mathrm{deg}]$ & $75^{\circ}$ & $80^{\circ}$ \\
\hline & Inlet relative flow angle, $\beta_{2}[\mathrm{deg}]$ & $-30^{\circ}$ & $-20^{\circ}$ \\
\hline & Outlet absolute flow angle, $\alpha_{3}[\mathrm{deg}]$ & $0^{\circ}$ & $0^{\circ}$ \\
\hline & Outlet relative flow angle (RMS), $\beta_{3, R M S}[\mathrm{deg}]$ & $-65^{\circ}$ & $-65^{\circ}$ \\
\hline & Inlet radius, $r_{2}[\mathrm{~mm}]$ & 50.39 & 17.09 \\
\hline & Hub-to-tip radius, $r_{3 h} / r_{3 s}[-]$ & 0.40 & 0.40 \\
\hline & Relative velocity ratio, $W_{R}[-]$ & 2.15 & 2.61 \\
\hline & Diameter ratio, $d_{2} / d_{3, R M S}[-]$ & 1.922 & 2.40 \\
\hline & Flow coefficient, $\phi[-]$ & 0.25 & 0.20 \\
\hline & $\mathrm{N}^{\circ}$ blades, $z[-]$ & 14 & 18 \\
\hline & Blade thickness, $t[\mathrm{~mm}]$ & 0.50 & 0.50 \\
\hline & Inlet blade height, $b_{2}[\mathrm{~mm}]$ & 5.70 & 2.15 \\
\hline & Tip clearance, $c[\mathrm{~mm}]$ & 0.1 & 0.1 \\
\hline \multirow{3}{*}{ Spiral casing } & Inlet centroid radius, $r_{l}[\mathrm{~mm}]$ & 61.73 & 21.58 \\
\hline & Inlet Area, $A_{I}\left[\mathrm{~mm}^{2}\right]$ & 468.90 & 43.71 \\
\hline & Aspect ratio, $A R[-]$ & 1.23 & 1 \\
\hline
\end{tabular}

\subsection{Performance parameters}

The parameters used to quantify the performance of the turbines are listed as follows:

- total-to-static isentropic efficiency $\eta_{\text {is,total-to-static }}$ : 


$$
\begin{gathered}
\eta_{\text {is,total-to-static }}=\frac{\left(h_{\text {in }}^{0}-h_{\text {out }}\right)}{\left(h_{\text {in }}^{0}-h_{\text {is,out }}\right)} \\
h_{\text {tot }, \text { in }}=f\left(p_{\text {in }}^{0}, T_{\text {in }}\right) \\
h_{\text {is,out }}=f\left(p_{\text {out }}, s_{\text {in }}^{0}\right) \\
s_{\text {tot }, \text { in }}=f\left(p_{\text {in }}^{0}, T_{\text {in }}\right) \\
h_{\text {tot }, \text { in }}=f\left(p_{\text {out }}, T_{\text {out }}\right)
\end{gathered}
$$

where $h$ and $s$ are the specific enthalpy and entropy, respectively, (in $[\mathrm{kJ} / \mathrm{kg}]$ and $[\mathrm{kJ} / \mathrm{kgK}]$ ).

- pressure ratio $r_{p}$ :

$$
r_{p}=\frac{p_{\text {in }}^{0}}{p_{\text {out }}}
$$

- blade speed ratio $\frac{u}{c_{0}}$ :

$$
\begin{gathered}
\frac{u}{C_{0}}=\frac{u}{\sqrt{2 * \Delta h_{i s}}} \\
\Delta h_{i s}=h_{\text {in }}^{0}-h_{\text {is,out }}
\end{gathered}
$$

where $u[\mathrm{~m} / \mathrm{s}]$ is the tip speed of the runner and $\Delta h_{i s}[\mathrm{~kJ} / \mathrm{kg}]$ is the isentropic specific work.

\section{Instruments}

This Section describes the numerical tools used to achieve the aims of this paper. The first of the following two subSections describes the features of the benchmark CFD approach and comments the results of the simulations conducted on the two turbine designs. The second sub-Sections shows the simulation setups of MULTALL that implements the proposed modelling approach.

\subsection{Turbine models solved by STAR-CCM+}

The reliability of the proposed modelling technique is checked against the results of computations performed on a 360degree domain of the entire turbine (including the clearance at the blade tip), using the state-of-the-art commercial CFD code Siemens STAR-CCM+ ver. 14.02.010-R8. This CFD model is conceived as benchmark for preliminary design calculations: grid size, physical models and turbulence closure have been therefore chosen as trade-off between accuracy and computation time. In accordance with the scope of the model, the overall complexity has been limited to the level that allows fulfilling the convergence criterion (residuals lower that 10-5 for all the transport equations solved with the segregated enthalpy-based approach [23]) in a computation time not exceeding $90 \mathrm{~min}$ on an Intel(R) Core(TM) i7-5500U CPU@2.40GHz computer). The Multi-Reference-Frame MRF steady state approach has been selected as motion model in conjunction with the "frozen rotor" interface sub-model to manage the data exchange from moving (runner) and fixed (casing) regions. The frozen rotor approach has been preferred to the mixing-plane because it predicts global turbine performance at design almost equal to those obtained using the mixing-plane approach (see Fig.4) and, it is supposed that at off-design it estimates losses due to wakes and uneven flow distribution always less optimistic than those predicted by the mixing-plane approach (although frozen rotor predictions are very dependent on the relative position between the rotor and the spiral casing tongue in this case). Uniform total-pressure-inlet and static-pressure-outlet were used as boundary conditions of the simulations. With regard to the computational grid, it is worth considering that the grid sensitivity study loses the physics coherence when the mesh refinement exceeds the grid density corresponding to the uniform distribution of the cell sizes in the entire domain - included the near-wall region - in which $\mathrm{y}^{+}$at the near-wall cells reached the minimum value consistent with the selected near-wall treatment. Of course, in high Reynolds number RANS calculations, this maximum grid density is usually by far denser than the grid that allows obtaining results not affected by the cell size. However, this could not be the case when low-Reynolds number calculations are dealt with. As a matter of fact, the 360 degree domain of the NS03 design (averaged rotor channel Reynolds number $R_{3, a v g}=$ $\frac{w_{3, a v g} * D h_{3}}{v_{3, a v g}}=16000$ calculated at the channel outlet $-D h_{3}$ is the channel hydraulic diameter), requires approximately 600000 cell numbers (polyhedral cell core and 2-prism near-wall layer) to obtain uniform cell size in the entire domain and a wall $\mathrm{y}^{+}$everywhere approximately equal to 12 . Since the Spalart-Allmaras one-equation turbulence model coupled with the standard wall function approach permits to solve the steady-state RANS equations on the above-described grid with a computation time close to the maximum allowed, this grid has been chosen as the final grid for all the 360-degree domain computations. Note that, from the numerical point of view, the results of these computations are still dependent on the grid size. Accordingly, the NS05 design $\left(R_{3, \text { avg }}=370000\right)$ could admit computations on a more refined grid if the computation time constraint is relaxed, as clearly demonstrated by the cell size distribution visible in wireframe views of the of the NS05 final grid reported in Figure 3. Finally, all the calculations were split in two steps: the first was 
performed considering constant density gas until the constrain on the residual was achieved; the second step was a restarted calculation (from the flow field solution obtained in the previous step) considering ideal gas properties. Table 3 summarizes the main features of the simulation setup for the two turbomachine designs considered in this work.

Tab. 3 - CFD setup used in the simulations performed with star CCM+

\begin{tabular}{|c|c|c|c|}
\hline \multirow{2}{*}{$\begin{array}{c}\text { RANS - turbulence } \\
\text { closure }\end{array}$} & Turbulence model & Near-wall treatment & Wall y+ \\
\cline { 2 - 4 } & $\begin{array}{c}\text { Standard Spalart- } \\
\text { Allmaras [24] }\end{array}$ & $\begin{array}{c}\text { Logarithmic wall } \\
\text { function }\end{array}$ & $>12$ \\
\hline $\begin{array}{c}\text { Domain grid density } \\
\text { (grid points) }\end{array}$ & Casing & Runner & Total \\
\cline { 2 - 4 } & $200 \mathrm{k}$ & $400 \mathrm{k}$ & $600 \mathrm{k}$ \\
\hline
\end{tabular}
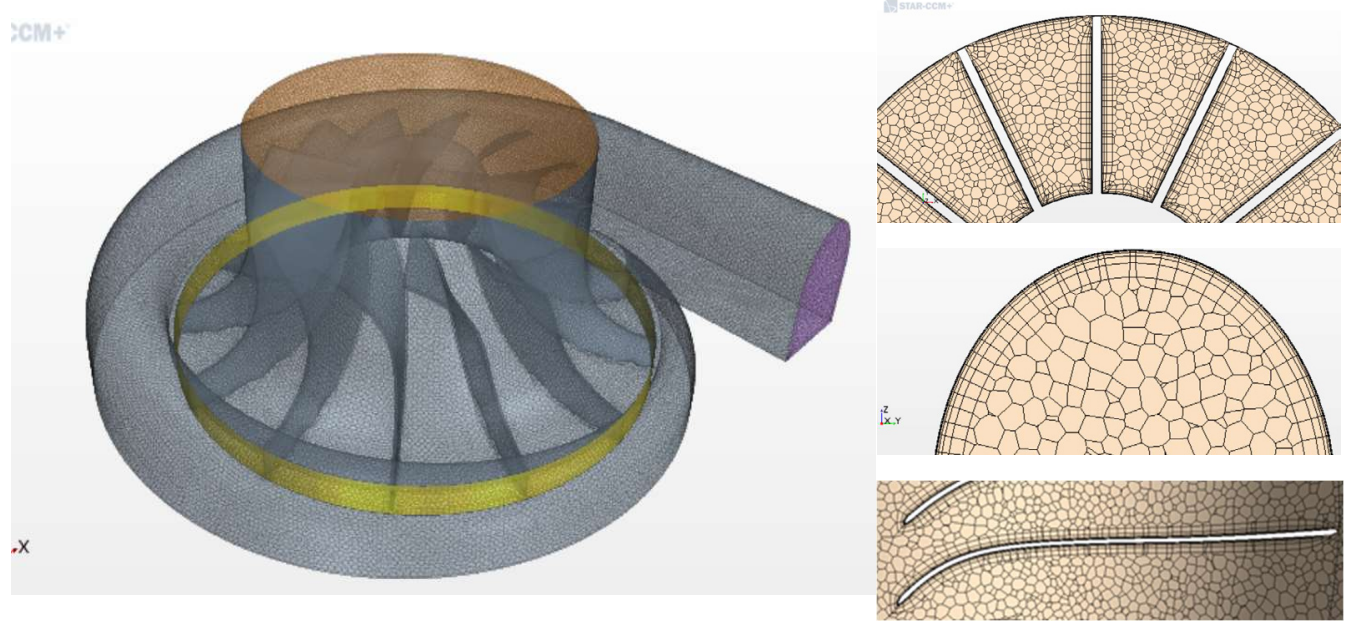

Fig. 3 - Computational grid details of NS05 design model in star CCM+.

Figure 4 shows the curves of total-to-static isentropic efficiency against the tip-speed ratio and the dimensionless massflowrate against the expansion ratio for the two turbine designs considered here as predicted by the Star CCM+® models just described.
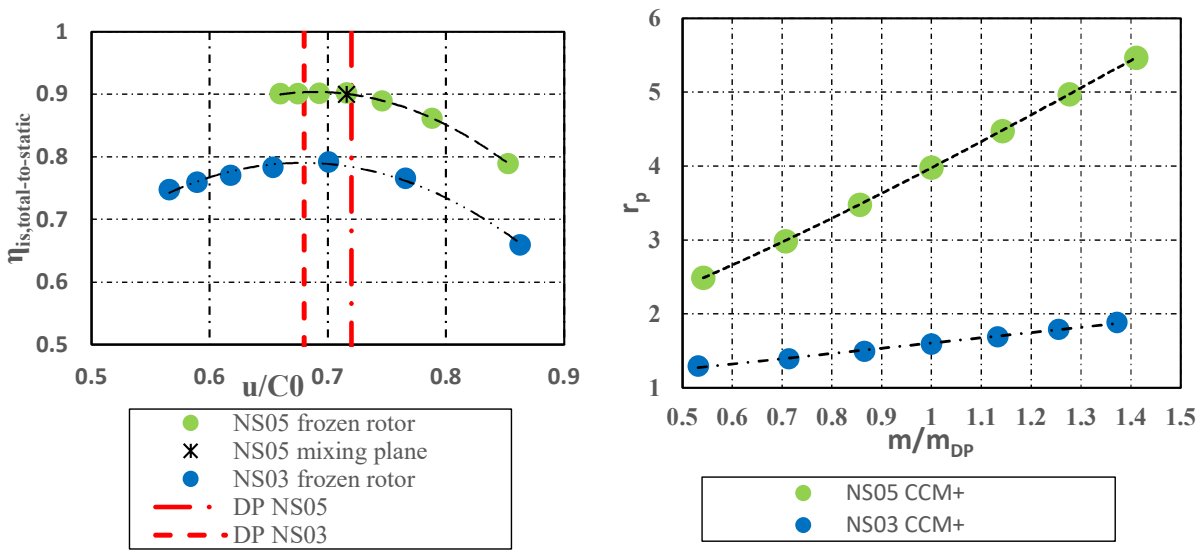

Fig. 4 - Total to static isentropic efficiency vs tip speed ratio curve (left) and expansion ratio vs m/mDP (right) of NS05 and NS03 designs predicted by star $\mathrm{CCM}+$.

The CFD predictions show that:

a) The CFD models solved by the state-of-the-art CFD code star CCM+ predict a reasonable trend of the total-tostatic isentropic efficiency;

b) The efficiency and the mass-flow rate predicted at the best efficiency duty for each design well-matches the corresponding values expected from the preliminary design procedure [21, 22], as further validation of its wellacknowledged reliability. 
Note that the Reynolds number of the NS05 machine is noticeably higher than that of the NS03 design. As discussed in the Results section, it is expected that a Low-Reynolds wall treatment can strongly modify the prediction of the NS03 turbine performance as obtained from the high-Reynolds approach presented above.

\subsection{Turbine models solved by MULTALL}

The 3D single-blade passage including the clearance at the blade tip and the spiral volute model described in detail in Section 2 has been implemented in MULTALL. A fully structured grid with near wall refinement has been used to mesh the entire domain. The grid density used in MULTALL is equivalent to the one set up in star-CCM+. Also, the near-wall grid clustering has been defined to achieve a minimum wall $\mathrm{y}+$ value approximately equal to 12 as per Star-CCM + . Thus, once selected the one-equation Spalart-Allmaras [24] turbulence closure implemented in MULTALL, a fair comparison between all the CFD models used in this paper is allowed. Note that also low-Reynolds calculations have been performed with MULTALL because the single channel CFD model allows to reach an acceptable computation time (<90min) even considering the additional grid refinement required to extend the application of the Spalart-Allmaras turbulence closure up to the wall. In this last case, the near wall region features the grid refinement that allows for a minimum wall $y+$ value equal to 0.5. Table 4 summarizes the main features of the NS05 and NS03 turbine models, while Fig. 5 shows some details of the computational domain of the NS05. To include the vaneless spiral volute casing model, the boundary conditions described in Section 2 were applied to solve the steady-state RANS equations [11]. At the end of the calculations, each computation reached values of the average residual (defined as the average percentage change in velocity per time step divided by the RMS velocity of all grid points) and continuity error lower than 0.001 and 0.01 , respectively.

Tab. 4 - CFD setup used in the simulations performed with MULTALL

\begin{tabular}{|c|c|c|c|c|c|}
\hline \multirow{3}{*}{$\begin{array}{l}\text { Physical models and } \\
\text { boundary conditions }\end{array}$} & Turbulence model & Near-wall treatment & Wall $\mathbf{y}^{+}$ & Imposed inl & yaw angle $\alpha$ \\
\hline & \multirow{2}{*}{$\begin{array}{c}\text { Spalart-Allmaras } \\
{[24]}\end{array}$} & Standard High-Re wall function & $>12$ (coarse) & \multirow{2}{*}{$4.3-\mathrm{NS} 05$} & \multirow{2}{*}{4.48 - NS03 } \\
\hline & & - & $>0.5$ (refined) & & \\
\hline \multirow{3}{*}{$\begin{array}{l}\text { Domain grid density } \\
\text { (grid points) }\end{array}$} & TP & Runner & Downstream & Total & Grid ID \\
\hline & $23 \times 18 \times 16^{\S}$ & $23 \times 18 \times 45^{\xi}$ & $23 \times 18 \times 8^{\S}$ & $\sim 28 \mathrm{k}$ & Coarse \\
\hline & $46 \times 37 \times 32^{\S}$ & $46 \times 37 \times 90^{\S}$ & $46 \times 37 \times 16^{\S}$ & $\sim 235 \mathrm{k}$ & Refined \\
\hline
\end{tabular}
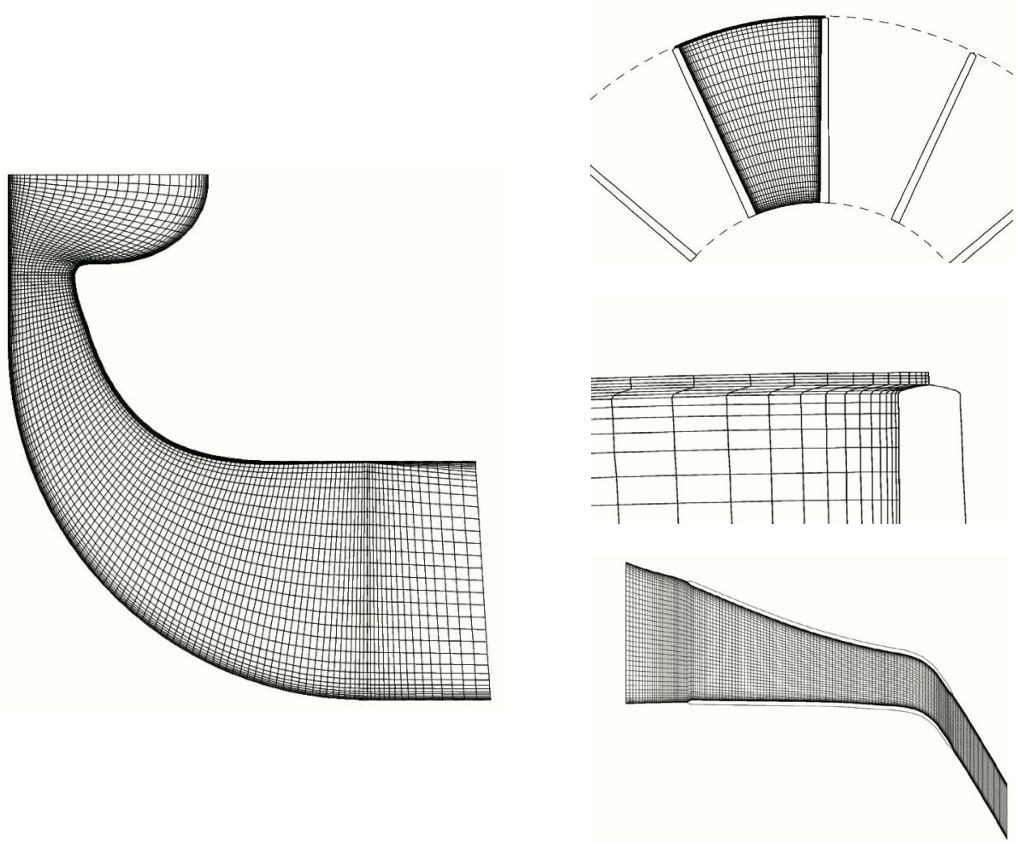

Fig. 5 - Computational grid details of NS05 design model in MULTALL. 


\section{Results}

The results of the simulations conducted with the two CFD approaches are compared to each other in the following. Figures 6 and 7compare the efficiency and performance curves resulting from the CFD calculations of the turbine designs NS05 and NS03, respectively.
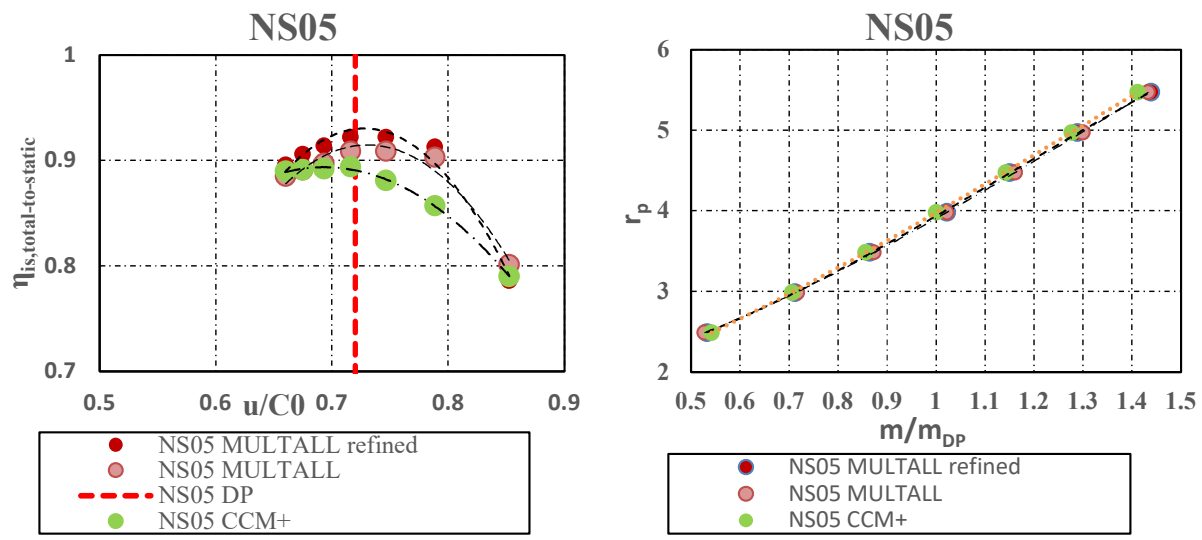

Fig. 6 -Isentropic efficiency curves (left) and mass-flowrates curves (right) of the NS05 design as predicted by MULTALL and star $\mathrm{CCM}+$.
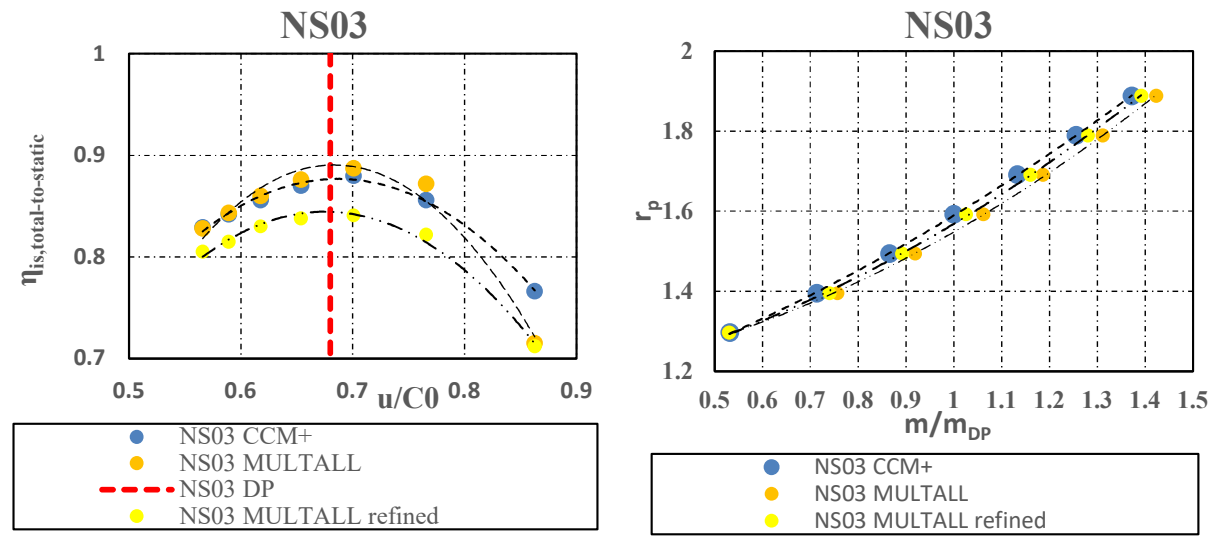

Fig. 7 - Isentropic efficiency curves (left) and mass-flowrate curves (right) of the NS03 design as predicted by MULTALL and star $\mathrm{CCM}+$.

The spiral casing modelling approach implemented in MULTALL was able to predict a reasonable trend of the total-tostatic isentropic efficiency curves for both the turbine designs. Moreover, the results from MULTALL are slightly affected by the grid refinement and the extension of the turbulence model up to the wall in the case of NS05 turbine, for which the refined grid results have been probably achieved the independency on the grid density. In fact, the moderate efficiency increase obtained from the calculations performed on the refined grid in the NS05 turbine is compatible with an improved resolution of the high-Re flow developing within that turbine design due to the increase of the grid density in the bulk flow region, whereas the solution of the boundary layer does not modify noticeably the wall shear stress obtained from the wall function in the computations performed on the coarse grid. In contrast, the strong efficiency decrease obtained from the refined grid calculations in the NS03 turbine can be tentatively attributed to the improved resolution of the lowRe flow developing in that turbine design in the boundary layer surroundings and, differently from the NS05 case, it is not possible to discuss about the dependency of the results on the grid density because the refined and coarse grid results depend on the difference existing in both the equations solved and the grid density. Further investigations are needed to confirm this discussion, however, assuming its consistency, its outcomes can be extended to the CCM+ models under the hypothesis that grid refinements have the same effects on the predictions of models featuring equal cell density, equal turbulence model and different grid structure (hexahedral vs polyhedral). Accordingly, a fair comparison between the two CFD approaches (MULTALL and $\mathrm{CCM}+$ ) must be performed considering the results obtained on the coarse grid, and noting that the MULTALL predictions can be assumed as numerically validated (i.e., grid independent) only in the NS05 case. Hence, with reference to the coarse grid results, the mass-flow rate curve predicted by MULTALL for the NS05 design is in very good agreement with the one predicted by star-CCM+. MULTALL always overestimates the predictions 
of CCM + by approximately $2 \%$. On the other hand, the difference between the efficiency predicted by the two models ranges between $0 \%$ and $5 \%$ and MULTALL always overestimates the $\mathrm{CCM}+$ predictions. Considering the coarse grid predictions of the NS03 performance, both the mass-flowrate and the efficiency values are overpredicted by MULTALL (by approximately $+5 \%$ the former and by up to $+2 \%$ the latter, with the only exception for the highest tip-speed ratio where MULTALL underestimates the efficiency predicted by CCM+ by approximately $-7 \%$ ). In summary, MULTALL tends to overestimate the mass flowrate and total-to-static isentropic efficiency if compared to the benchmark model. However, the deviation between the two predictions is generally limited between $0 \%$ to $5 \%$. Since the benchmark and single channel models feature the same turbulence closure and equivalent grid density, to a first approximation, the differences in the performance predictions could be attributed mainly to the equivalent spiral casing model proposed in this work. It is likely that the spiral casing modelling approach suggested here provides a more uniform flow distribution at the inlet of the runner and slightly underestimates the wall friction losses of the spiral casing compared to the actual one (see Section 2). Both these phenomena can contribute to increase the efficiency of the turbines and also to make them able to process a larger amount of working fluid.

It is opinion of the authors that the discrepancies in the performance predictions discussed above are acceptable for preliminary design calculations. Thus, these results assess the effectiveness of this novel modelling approach.

The comparison between the computation time required by the two codes shows that MULTALL takes about the $30 \%$ of the computation time required by STAR-CCM+ at equal grid density (approximately 5 min against $15 \mathrm{~min}$ ). On the other hand, it is worth considering that the spiral casing modelling approach implemented in MULTALL also allows performing Low-Re computations of the NS03 design, which cannot be completed in a computation time reasonable for preliminary design purpose by using the traditional 360-degree modelling approach in Star CCM+ (because of the noticeable increase in the cell numbers required by such computations). Finally, it is worth mentioning that the spiral casing modelling approach proposed in the present paper should be suited to application with any CFD code (however, the authors were not able to obtain converged solutions for single blade channel CFD calculations performed with STAR-CCM+ when the yaw angle specified at the total pressure inlet boundary decreases up to the required value).

\section{Conclusions}

A novel approach to model the vaneless spiral casings of radial inflow turbines in single-channel CFD simulations was applied to two turbine designs using the open-source CFD code MULTALL. The aerodynamic performance of the turbines so obtained were validated against the results of CFD calculations performed with the commercial code STAR$\mathrm{CCM}+$ on 360-degree computational domains. The results showed that:

- The trends of the mass-flowrate and efficiency curves calculated by MULTALL and STAR CCM+ for the two turbines are in good agreement. In the entire range of pressure ratios considered for the comparison, MULTALL never exceeded $\mathrm{a}+5 \%$ overestimation of the STAR CCM + predictions.

- The difference of the efficiency and processed mass-flowrate obtained in the MULTALL and CCM+ calculations are due mainly to the spiral casing model because the setups of the simulations feature the same turbulence closure and equivalent grid density, whereas the proposed model of the spiral casing unavoidably forces the even distribution of the flow between all the blade and imposes a slight underestimation of the spiral casing wall friction losses.

- MULTALL is able to perform single blade passage 3D simulations with computation time $300 \%$ shorter than fully 360 degrees simulations performed with $\mathrm{CCM}+$ on grids having equal density.

This leads to conclude that:

- The validity and the limitations of the proposed modelling approach for spiral casings are assessed.

- MULTALL coupled with the novel modelling approach to simulate spiral volute casings can be employed as a fast tool to support the preliminary design of radial inflow turbines.

\section{Nomenclature}

$\begin{array}{ll}\mathrm{P} & \text { pressure, } \mathrm{Pa} \\ \mathrm{T} & \text { temperature, }{ }^{\circ} \mathrm{C} \\ \dot{m} & \text { mass flow rate, } \mathrm{kg} / \mathrm{s} \\ \mathrm{V} & \text { velocity, } \mathrm{m} / \mathrm{s} \\ \mathrm{V} & \text { voltage, } \mathrm{V} \\ \mathrm{A} & \text { area, } \mathrm{m}^{2} \\ \mathrm{~s} & \text { entropy, } \mathrm{kJ} / \mathrm{kgK} \\ \mathrm{h} & \text { enthalpy, } \mathrm{kJ} / \mathrm{kg} \\ \mathrm{r}_{\mathrm{p}} & \text { pressure ratio }\end{array}$

$\begin{array}{ll}\mathrm{u} & \text { tip speed, } \mathrm{m} / \mathrm{s} \\ \mathrm{C}_{0} & \text { isentropic gas speed, } \mathrm{m} / \mathrm{s} \\ \mathrm{rpm} & \text { revolutions per minute } \\ \text { Greek symbols } \\ \eta & \text { efficiency } \\ \rho & \text { density, } \mathrm{kg} / \mathrm{m}^{3} \\ \text { Subscripts } \\ \text { stat } \quad \text { static } \\ \text { in } & \text { inlet }\end{array}$

$\begin{array}{ll}\text { out } & \text { outlet } \\ \text { corr } & \text { corrected } \\ \text { ref } & \text { reference } \\ \text { tot } & \text { total } \\ \text { dyn } & \text { dynamic } \\ \text { is } & \text { isentropic } \\ \text { mecc } & \text { mechanical } \\ \text { max } & \text { maximum }\end{array}$




\section{References}

[1] Simpson, A. T., Spence, S. W. T., and Watterson, J. K. April 9, 2009. "A Comparison of the Flow Structures and Losses Within Vaned and Vaneless Stators for Radial Turbines." ASME. J. Turbomach. July 2009; 131(3): 031010.

[2] Descombes, G., Maroteaux, F., Moreno, N., Jullien, J. March 2003. "Analysis of energy conversion within a radial turbine stage", Int.J. Thermodynamics, Vol. 6 (No.1), pp. 41-48.

[3] Hellström, F., Fuchs, L. (2008). "Numerical computations of pulsatile flow in a turbo-charger". AIAA-2008-073, 46th AIAA paper.

[4] Odabaee, M., Shenechi, M., Hooman, K. December 2014. CFD Simulation and FE Analysis of a High Pressure Ratio Radial Inflow Turbine. 19th Australasian Fluid Mechanics Conference Melbourne, Australia 8-11.

[5] ANSYS, Inc. (2016) ANSYS Fluent User's Guide, Release 17.2.

[6] Denton, J.D., 1978, "Throughflow Calculations For Transonic Axial Flow Turbines", J Eng Power Trans ASME, Volume 100, Issue 2, Apr, Pages 212-218.

[7] Denton, J.D., July 1983, "An improved time-marching method for turbomachinery flow calculation", Journal of Engineering for Gas Turbines and Power, Volume 105, Issue 3, Pages 514-521.

[8] He, L., Denton, J.D., July 1994, "Three-dimensional time-marching inviscid and viscous solutions for unsteady flows around vibrating blades", Journal of Turbomachinery, Volume 116, Issue 3, Pages 469-476.

[9] Denton, J.D., Xu, L., 1999, “The exploitation of three-dimensional flow in turbomachinery design", Proceedings of the Institution of Mechanical Engineers, Part C: Journal of Mechanical Engineering, Volume 213, Issue 2, Pages 125-137.

[10] Denton, J.D., 1990, "Calculation of three-dimensional viscous flow through multistage turbomachines", American Society of Mechanical Engineers, Pages GT19 10p.

[11] Denton, J.D., 1 December 2017, "Multall-an open source,computational fluid dynamics based, turbomachinery design system", Journal of Turbomachinery, Volume 139, Issue 12, Article number 121001.

[12] Denton, J.D., Dawes, W.N., 1999, “Computational fluid dynamics for turbomachinery design”, Proceedings of the Institution of Mechanical Engineers, Part C: Journal of Mechanical Engineering. Volume 213, Issue 2, Pages 107124.

[13] Pullan, G., Denton, J.D., Dunkley, N., April 2003, “An experimental and computational study of the formation of a streamwise shed vortex in a turbine stage", Journal of Turbomachinery, Volume 125, Issue 2, Pages 291-297.

[14] Rosic, B., Denton, J.D., Pullan, G., October 2006, "The importance of shroud leakage modeling in multistage turbine flow calculations", Journal of Turbomachinery, Volume 128, Issue 4, Pages 699-707.

[15] Kaschel, C.E., Denton, J.D., April 2006, "Experimental and numerical investigation of the unsteady surface pressure in a three-stage model of an axial high pressure turbine", Journal of Turbomachinery, Volume 128, Issue 2, Pages 261-272.

[16] Crichton, D., 2007, "Fan Design and Operation for Ultra Low Noise", PhD dissertation, University of Cambridge, Engineering Department.

[17] Denton, J.D., 2010, "Some limitations of turbomachinery CFD", Proceedings of the ASME Turbo Expo, Volume 7, Issue PARTS A, B, AND C, Pages 735-745.

[18] Persson, M., June 2015, "Highly loaded HPT blading in KTH test turbine", Thesis for the degree of Master of Science in Engineering, Division of Thermal Power Engineering Department of Energy Sciences Lund University, Sweden.

[19] Danieli, P., Masi, M., Delibra, G., Corsini, A., Lazzaretto, A. June 2020. “Assessment of Multall as CFD code for the analysis of tube-axial fans. Proceedings of ASME Turbo Expo 2020, Turbomachinery Technical Conference and Exposition, GT2020, June 22-26, London, England. Paper GT2020-14953.

[20] Whitfield, A., Baines, N. C. 1990. "Design of radial turbomachines”. Harlow, Essex, England, Longman Scientific \& Technical.

[21] Chappie, P. M., Flynn, P. F., and Mulloy, J. M. January 1, 1980. "Aerodynamic Design of Fixed and Variable Geometry Nozzleless Turbine Casings." ASME. J. Eng. Power. January 1980; 102(1): 141-147.

[22] Cordier, O. 1953. “Ähnlichkeitsbedingungen für Strömungsmaschinen”. In:BWK Bd6.

[23] Van Doormaal, J. P., Raithby, G. D., and McDonald, B. H. April 1, 1987. "The Segregated Approach to Predicting Viscous Compressible Fluid Flows." ASME. J. Turbomach. April 1987; 109(2): 268-277.

[24] Spalart, P. R., Allmaras, S. R. 1992. "A One-Equation Turbulence Model for Aerodynamic Flows", AIAA, Paper 92-0439. 